\title{
A review of research trends in physiological abnormalities in autism spectrum disorders: immune dysregulation, inflammation, oxidative stress, mitochondrial dysfunction and environmental toxicant exposures
}

\author{
DA Rossignol ${ }^{1}$ and RE Frye ${ }^{2}$ \\ ${ }^{1}$ International Child Development Resource Center, Melbourne, FL, USA and ${ }^{2}$ Arkansas Children's Hospital Research \\ Institute, University of Arkansas for Medical Sciences, Little Rock, AR, USA
}

\begin{abstract}
Recent studies have implicated physiological and metabolic abnormalities in autism spectrum disorders (ASD) and other psychiatric disorders, particularly immune dysregulation or inflammation, oxidative stress, mitochondrial dysfunction and environmental toxicant exposures ('four major areas'). The aim of this study was to determine trends in the literature on these topics with respect to ASD. A comprehensive literature search from 1971 to 2010 was performed in these four major areas in ASD with three objectives. First, publications were divided by several criteria, including whether or not they implicated an association between the physiological abnormality and ASD. A large percentage of publications implicated an association between ASD and immune dysregulation/inflammation (416 out of 437 publications, $95 \%$ ), oxidative stress (all 115), mitochondrial dysfunction (145 of $153,95 \%$ ) and toxicant exposures (170 of $190,89 \%$ ). Second, the strength of evidence for publications in each area was computed using a validated scale. The strongest evidence was for immune dysregulation/ inflammation and oxidative stress, followed by toxicant exposures and mitochondrial dysfunction. In all areas, at least $45 \%$ of the publications were rated as providing strong evidence for an association between the physiological abnormalities and ASD. Third, the time trends in the four major areas were compared with trends in neuroimaging, neuropathology, theory of mind and genetics ('four comparison areas'). The number of publications per 5-year block in all eight areas was calculated in order to identify significant changes in trends. Prior to 1986, only 12 publications were identified in the four major areas and 51 in the four comparison areas (42 for genetics). For each 5-year period, the total number of publications in the eight combined areas increased progressively. Most publications (552 of $895,62 \%)$ in the four major areas were published in the last 5 years (2006-2010). Evaluation of trends between the four major areas and the four comparison areas demonstrated that the largest relative growth was in immune dysregulation/inflammation, oxidative stress, toxicant exposures, genetics and neuroimaging. Research on mitochondrial dysfunction started growing in the last 5 years. Theory of mind and neuropathology research has declined in recent years. Although most publications implicated an association between the four major areas and ASD, publication bias may have led to an overestimation of this association. Further research into these physiological areas may provide insight into general or subset-specific processes that could contribute to the development of ASD and other psychiatric disorders.

Molecular Psychiatry (2012) 17, 389-401; doi:10.1038/mp.2011.165; published online 6 December 2011
\end{abstract}

Keywords: autism; immune dysregulation; inflammation; oxidative stress; mitochondrial dysfunction; environmental toxicants

\section{Introduction}

Autism spectrum disorders (ASD) are a heterogeneous group of neurodevelopmental disorders that are defined by behavioral observations, and are charac-

Correspondence: Dr DA Rossignol, International Child Development Resource Center, 3800 West Eau Gallie Blvd., Melbourne, FL, 32934, USA.

E-mail: rossignolmd@gmail.com

Received 11 May 2011; revised 20 October 2011; accepted 8 November 2011; published online 6 December 2011 terized by impairments in communication and social interaction along with restrictive and repetitive behaviors. ${ }^{1}$ ASD includes autistic disorder, Asperger syndrome, pervasive developmental disorder-not otherwise specified and Rett syndrome. An estimated 1 out of 110 individuals in the US is currently affected with an ASD. ${ }^{2}$ The etiology of ASD is unclear at this time. Although several genetic syndromes, such as Fragile X and Rett syndromes, have been associated with ASD, empirical studies have estimated that genetic syndromes only account for $6-15 \%$ of ASD 
cases. $^{3}$ Therefore, the majority of ASD cases are not due to a simple single gene or chromosomal disorder. Although many of the cognitive and behavioral features of ASD are thought to arise from dysfunction of the central nervous system (CNS), evidence from many fields of medicine has documented multiple non-CNS physiological abnormalities associated with ASD, ${ }^{4-7}$ suggesting that, in some individuals, ASD arises from systemic, rather than organ-specific, abnormalities. Specifically, in recent decades, research and clinical studies have implicated physiological and metabolic systems that transcend specific organ dysfunction, such as immune dysregulation, inflammation, impaired detoxification, environmental toxicant exposures, redox regulation/ oxidative stress and energy generation/mitochondrial systems. ${ }^{8}$ In this context, ASD may arise from, or at least involve, systemic physiological abnormalities rather than being a purely CNS disorder, ${ }^{9}$ at least in a subset of individuals with ASD.

Interestingly, this view of ASD has similarities with recent studies in other psychiatric disorders. Like ASD, a simple single gene or chromosomal abnormality has not been found to explain most psychiatric disorders. Despite the fact that linkage studies have identified candidate regions of certain chromosomes that could be associated with many psychiatric disorders, studies have been inconsistent. For example, genetic polymorphisms have been associated with susceptibility to psychiatric disorders such as schizophrenia, but most polymorphisms identified are in the non-coding regions of the genome, making the understanding of how these genetic changes contribute to psychiatric disorders opaque. ${ }^{10,11}$ Research studies in a wide variety of psychiatric disorders, including ASD, have started to investigate gene-environment interactions and epigenetic factors, rather than fixed genetic defects. Other research studies examining the etiology of psychiatric disorders have embraced the study of pathophysiological mechanisms that could more directly result in cellular dysfunction and the subsequent development of psychiatric disorders. Like ASD, pathophysiological mechanisms identified in some psychiatric disorders include immune dysregulation, inflammation, impaired detoxification, environmental toxicant exposures, redox regulation/oxidative stress and mitochondrial dysfunction. ${ }^{12-15}$

Immune dysregulation and inflammation has been implicated in several psychiatric disorders. For example, neuroinflammation, which generally refers to CNS-specific, chronic glial reactions that may not demonstrate typical peripheral evidence of inflammation, has been implicated in several psychiatric disorders, ${ }^{16}$ including Alzheimer's disease, ${ }^{17}$ schizophrenia, ${ }^{18}$ bipolar disorder, ${ }^{19}$ and depression ${ }^{20}$ as well as ASD. ${ }^{21,22}$ Neuroinflammation can damage brain tissue through several mechanisms, including plaque formation, abnormal neuron growth, increased tau phosphorylation and proinflammatory cytokines release. ${ }^{16}$
Environmental toxicants exposure has been implicated in a wide variety of disorders. Toxicants, such as heavy metals, pesticides and chemicals, can damage cells by converging on similar biochemical pathways to produce adverse effects, such as increasing oxidative stress, depleting glutathione and impairing cellular signaling. ${ }^{23}$ Exposures to environmental toxicants, such as mercury, lead, arsenic, polychlorinated biphenyls and toluene, are known to cause neurodevelopmental disorders. ${ }^{24}$ Exposures to environmental toxicants have been implicated in certain psychiatric disorders, such as ADHD, ${ }^{25-27}$ depression $^{28}$ and schizophrenia ${ }^{29}$ as well as ASD. ${ }^{30-32}$

Oxidative stress is defined as damage to cellular tissue caused by free radicals such as reactive oxygen species. Oxidative stress has been implicated in a wide variety of disorders, including cardiovascular disease, $^{33}$ diabetes $^{34}$ and hypertension. ${ }^{35}$ Furthermore, oxidative stress has been implicated in several psychiatric disorders, ${ }^{13}$ including schizophrenia, ${ }^{36,37}$ depression, ${ }^{38}$ bipolar disorder ${ }^{39,40}$ and Alzheimer's disease. ${ }^{41}$ Oxidative stress has also been reported in some individuals with ASD. ${ }^{6,42}$

Finally, dysfunction in mitochondria, distinct cellular organelles that oxidize glucose and fatty acids to generate adenosine triphosphate, the energy carrier in most mammalian cells, ${ }^{43}$ has been implicated in several psychiatric disorders including schizophrenia, ${ }^{44-47}$ bipolar disorder, ${ }^{44-46}$ depression, ${ }^{45,46}$ dementia $^{45,46}$ as well as ASD.,48,49 In addition to energy production, mitochondria are intimately involved in programmed cell death (apoptosis), calcium homeostasis, synaptic plasticity and neurotransmitter release. ${ }^{50,51}$ Therefore, mitochondrial dysfunction can cause profound dysfunction of the CNS as well as other organ systems, particularly high-energy organs such as the gastrointestinal (GI) tract. ${ }^{4}$

The goal of this manuscript is to examine the evidence of these systemic physiological abnormalities in ASD. We have approached this in several ways. First, we analyzed trends in the number of publications in four specific areas of research over the past four decades, and compared these trends with four other areas of research in ASD involving nonphysiological abnormalities. Second, we have identified what percentage of studies in each of these research areas support these physiological mechanisms in ASD. Third, we rate the strength of these studies within each research area.

\section{Materials and methods}

\section{Search strategy}

A search of the PubMed, Google Scholar and SCOPUS databases from 1971 through 2010 was conducted to identify and collate pertinent publications using the search terms 'autism', 'autistic', 'ASD', 'Asperger', 'Rett', 'pervasive developmental disorder' and 'PDD' in all combinations with the terms described below in four separate literature reviews of: (1) inflammation and immune dysregulation; (2) oxidative stress; 
(3) mitochondrial dysfunction; and (4) environmental toxicant exposures. Publications of Rett syndrome were collated separately. To identify publications of inflammation or immune dysregulation, the following search terms were used: 'immune', 'immunology', 'immunological', 'autoimmune', 'autoimmunity', 'neuroinflammation', 'neuroinflammatory', 'inflammation', 'inflammatory', 'antibody', 'antibodies', 'immunoglobulin', 'infection', 'mucosal immunity', 'adaptive immunity', 'innate immunity', 'lymphocyte', 'glial activation', 'cytokines', 'prostaglandins', 'neopterin', ‘immunomodulatory', 'BDNF', 'allergies', 'asthma' and 'atopy.' To identify publications of oxidative stress, the following search terms were used: 'oxidized', 'oxidative', 'oxidant', 'oxidants', 'oxidation', 'oxidative stress', 'redox', 'glutathione', 'cysteine', 'antioxidant', 'antioxidants', 'peroxidation' and 'reactive oxygen.' To identify publications of mitochondrial dysfunction, the following search terms ${ }^{4}$ were used: 'mitochondria', 'mitochondrial', 'lactic', 'lactate', 'pyruvate', 'pyruvic', 'ammonia', 'creatine kinase', 'oxidative phosphorylation', 'phosphorylation', 'carnitine', 'acylcarnitine', 'fatty acid oxidation', 'alanine', 'respiratory chain', 'electron transport chain', 'energy', 'ATP' and 'adenosine.' Finally, to identify publications of environmental toxicant exposures, the following search terms were used: 'toxicant', 'toxin', 'metal', 'mercury', 'lead', 'chemical', 'pesticide', 'PCB', 'phthalate', 'solvent', 'pollutant', 'pollution', 'xenobiotic', 'bacteria', 'stress', 'antibiotic', 'fluoride', 'vitamin D', 'fatty acids', 'folate', 'family bereavement', 'delivery problem' and 'oxytocin.' For all four searches, a combined total of 1037 publications were identified. However, because we performed four separate literature searches, some publications were identified and placed into more than one category, although this number was relatively small (131 of 1037 publications or 13\%; see Supplementary Material for flow charts of literature searches, Supplementary Figures S1-S4). After consolidating these duplicated references, 906 unique publications discussing immune dysregulation, inflammation, oxidative stress, mitochondrial dysfunction or environmental toxicant exposures ('four major areas') in ASD were identified and these references are listed in the Supplementary Material.

In order to compare trends in research areas identified above with other well-known areas of ASD research, we performed separate literature searches for four additional areas ('four comparison areas') in ASD: neuroimaging, neuropathology, theory of mind and genetics. For neuroimaging, the following search terms were used: 'neuroimaging', 'MRI', 'magnetic resonance imaging', 'SPECT', 'single photon emission computed tomography', 'PET', 'positron emission tomography', 'fMRI', 'functional magnetic resonance imaging', 'MRS', 'magnetic resonance spectroscopy', 'MEG', 'magnetoencephalography', 'computed tomography' and 'diffusion tensor imaging.' For neuropathology, the following search terms were used: 'neuropathology', 'postmortem', 'autopsy' and 'necropsy', and for theory of mind: 'theory of mind' and 'ToM.' Finally, for genetics, the following search terms were used: 'gene', 'polymorphism', 'mutation', 'copy number variation', 'chromosomal deletion', 'chromosomal duplication' and 'genomic.' The flowcharts (Supplementary Figures S5-S8) and list of studies identified for each of these searches is available in the Supplementary material, and includes 654 unique publications for neuroimaging, 99 for neuropathology, 191 for theory of mind and 1576 for genetics. To determine the number of studies published on autism since 1971, a search of PUBMED from 1971 through 2010 using the search term 'autism' identified 16310 unique publications.

\section{Study selection}

Publications were initially included if they: (1) involved individuals or specimens from individuals with ASD or were animal models of ASD, and (2) reported at least one finding related to immune dysregulation, inflammation, oxidative stress, mitochondrial dysfunction or environmental toxicant exposures. Abstracts of identified publications were reviewed to determine if a publication should be included. If the abstract was obscure or missing, the publication was reviewed to determine if inclusion was warranted. Publications of ASD animal models were collated separately. Publications concerning the potential relationship between vaccine administration or vaccine ingredients (for example, thimerosal) and ASD that were identified by the literature searches were collated separately, and not included in the trend analysis because the majority did not present any unique data (many of these publications were editorials).

\section{Analysis strategy}

Publications were first grouped by the type of publication: clinical studies, review articles, hypothesis articles, treatment articles or animal models (Table 1). Clinical studies were defined as studies involving individuals with ASD or specimens from such individuals that produced unique outcome data but did not involve treatments. Review articles did not contain unique data but rather were reviews of other literature. Hypothesis papers were similar to review articles but were more speculative. Treatment articles involved a particular treatment for an abnormality. Animal models did not contain any human data. Secondly, publications were also grouped by whether they implicated or did not implicate an association between a particular physiological abnormality and ASD (Table 1). The number of identified publications per 5-year block was also calculated and this data is reported in Figure 1a. Because the range of the number of publications differed by several orders of magnitude across the different areas of research, we reported the number of publications ( $y$ axis) on a logarithmic scale. Figure 1b demonstrates the proportion of publications within each research area normalized by the total number of 

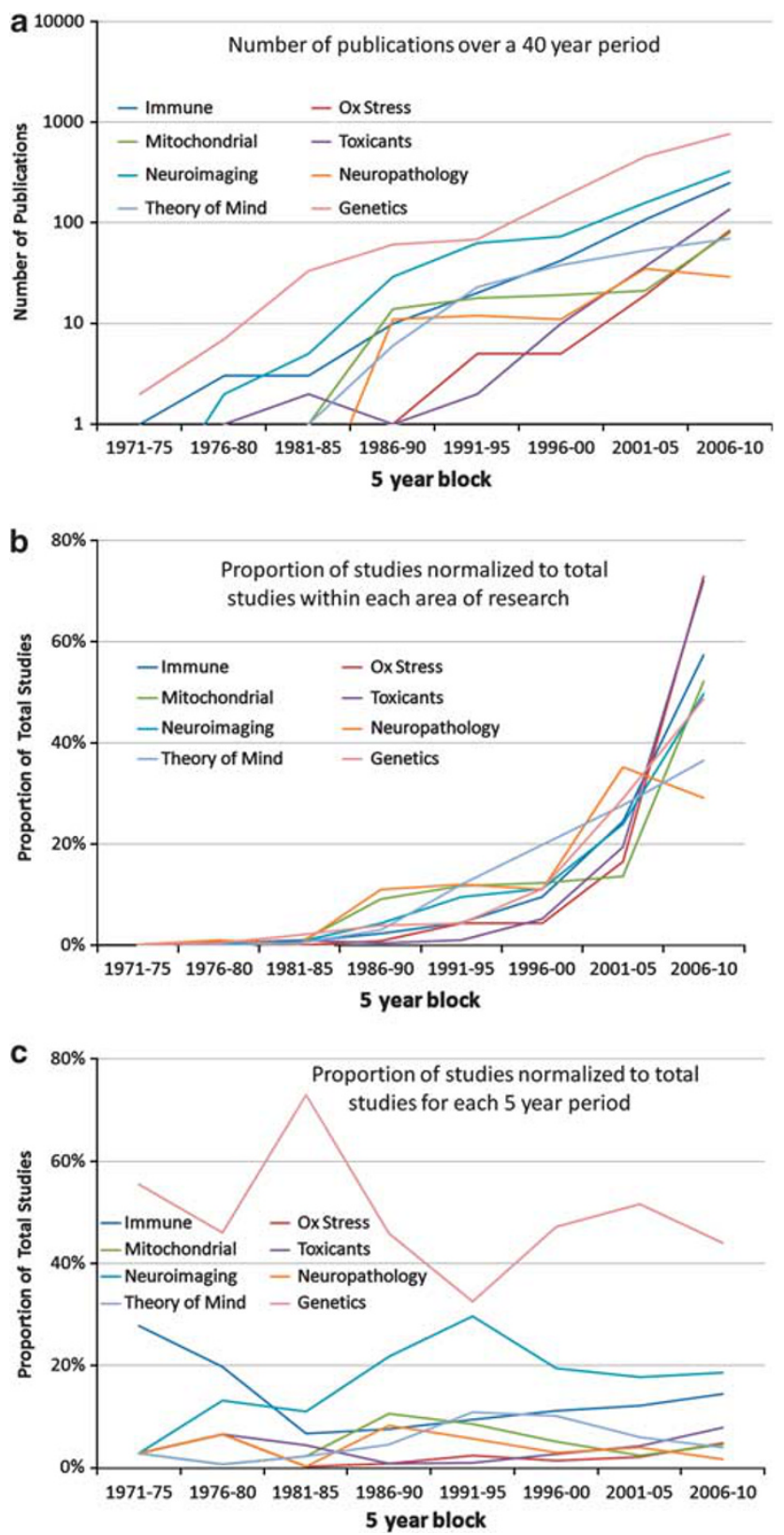

Figure 1 Graph of publications on autism spectrum disorder (ASD) by type and year of publication. (a) The number of publications in the eight areas per 5-year block on a logarithmic scale. (b) The proportion of publications within each research area normalized by the total number of publications within the specific research area. This allows the relative growth in each research area to be observed. (c) The proportion that each research area accounts for in the total number of publications identified for each specific 5 -year period. This allows the relative influence of each area of research to be determined over time.

publications within the specific research area. This allows the relative growth in each research area to be observed. Figure 1c demonstrates the proportion that each research area accounts for in the total number of publications identified for each specific 5-year period. This allows the relative influence of each area 
of research to be determined over time. Using $\chi^{2}$, we analyzed whether the number of studies differed across each research area within each 5-year block, if the number of studies changed over 5-year blocks within each research area and whether the change in the number of studies over 5-year blocks was different for different areas of research. Bonferroni correction was used to correct for multiple comparisons.

In order to assess the strengths and weaknesses of studies within the four major areas, all clinical and treatment studies were ranked using a validated level of evidence scale. ${ }^{52}$ Using this scale, each study was individually assessed to determine the corresponding level of evidence ('evidence level'), ranging from level 1 to 5. The highest evidence level (level 1) was assigned to a systematic review of RCTs (randomized controlled trials), an individual RCT (with narrow confidence intervals) or a cohort (prospective) study with good follow up; whereas the lowest evidence level (level 4 or 5) was assigned to case series or reports, or studies based on expert opinion without critical appraisal (Table 2). Using this scale, it was not possible to rank review articles, hypothesis articles or animal studies.

\section{Results}

\section{Publications identified by the search}

Four major areas. Of the 4422 initial publications (2033 duplicates) identified concerning immune dysregulation or inflammation in ASD, 532 met inclusion criteria (Supplementary Figure S1). Of the initial 1113 publications (623 duplicates) identified concerning oxidative stress in ASD, 115 met inclusion criteria (Supplementary Figure S2). Of the initial 1153 publications (360 duplicates) identified concerning mitochondrial dysfunction in ASD, 153 met inclusion criteria (Supplementary Figure S3). Finally, of the 3784 publications (834 duplicates) identified concerning environmental toxicant exposures in ASD, 237 met criteria for inclusion (Supplementary Figure S4).

Table 2 Levels of evidence (adapted from the Oxford 2011 Levels of Evidence ${ }^{52}$ )

Level Description

1 SR of RCT; individual RCT (with narrow confidence intervals); cohort (prospective) study with good follow -up

$2 \quad$ SR of cohort studies with homogeneity; individual cohort (prospective) study; low-quality RCT.

3 SR of case-control studies with homogeneity; individual case-control (retrospective) study.

$4 \quad$ Case series or reports.

5 Expert opinion without critical appraisal.

Abbreviations: RCT, randomized controlled trial, SR, systematic review.
Four comparison areas. For neuroimaging, out of 4807 initial publications (3291 duplicates), 654 met criteria for inclusion (Supplementary Figure S5). For neuropathology, out of 565 initial publications (245 duplicates), 99 met criteria for inclusion (Supplementary Figure S6). For theory of mind, out of 476 publications (81 duplicates), 191 met criteria for inclusion (Supplementary Figure S7). Finally for genetics, out of 11524 publications (6735 duplicates), 1576 met criteria for inclusion (Supplementary Figure S8).

Research trends in publications in four major areas Prior to 1986, there were almost no publications in the four major areas of physiological abnormalities in ASD. Specifically, prior to 1986, there were only seven publications discussing immune dysregulation or inflammation, one publication discussing oxidative stress, three publications discussing environmental toxicant exposures and one publication discussing mitochondrial dysfunction with reference to ASD. As seen in Figure 1a, since 1986, publications in the areas of immune dysregulation or inflammation have been the fastest growing with publications in environmental toxicant exposures growing second fastest. Research in immune dysregulation or inflammation started growing in the 1990s, whereas growth in research focusing on oxidative stress, mitochondrial dysfunction and environmental toxicant exposures started within the last 10 years. As growth in these areas of research started, there has been a precipitous increase in the number of publications on these subjects, with the majority of publications in these areas of research published within the last 5 years (2006-2010). For example, 57\% of the publications on immune dysregulation or inflammation, $73 \%$ of the publications on oxidative stress, $52 \%$ of the publications on mitochondrial dysfunction and $72 \%$ of the publications on environmental toxicant exposures in ASD have been published in the last 5 years.

Quantitative analysis of research trends in ASD in all research areas

Figure 1a provides a graphical representation of the number of papers published from 1971 to 2010, in 5 -year intervals. The number of publications is represented on a logarithmic scale to help visualization because of the 10-fold difference in the number of publications from the area with the least number of papers (neuropathology) to the area with the most number of papers (genetics). Overall, the total number of papers published for each research area was significantly different across research areas $\left(\chi^{2}(7)=4121, P<0.0001\right)$. Figure 1a demonstrates that the total number of papers published in genetics (1573) is almost equal to all other research areas combined (1839). The total number of papers on genetic research was 2.4 times more than the second most-studied research area (neuroimaging) and 3.6 times more than the third most-studied research area (immune). 
Overall, the total number of papers published per 5 -year period was significantly different across years $\left(\chi^{2}(7)=6029, P<0.0001\right)$. Figure 1a demonstrates that the total number of papers progressively increased for each 5-year interval from 1971-1975 to 2006-2010. The $\chi^{2}$ for the research area by year contingency table was significant $\left(\chi^{2}(49)=212, P<0.0001\right)$ indicating that the change in the number of papers across years was different for different research areas. This was primarily due to the fact that several areas of research demonstrated unique trends.

Figure $1 \mathrm{~b}$ demonstrates the trend in the number of publications published within each area of research (normalized to the total number of publications within the specific research area). Clearly, the research on oxidative stress and exposure to toxicants has grown the most as compared with all other research areas expect for immune (oxidative stress: mitochondrial dysfunction, $\chi^{2}(7)=24, \quad P<0.001$; neuropathology, $\chi^{2}(7)=43, \quad P<0.001$; genetics, $\quad \chi^{2}(7)=28, \quad P<0.001$; theory of mind, $\chi^{2}(7)=43, P<0.001$; neuroimaging, $\chi^{2}(7)=25, P<0.001$. toxicants: mitochondrial dysfunction, $\chi^{2}(7)=44, P<0.001$; neuropathology, $\chi^{2}(7)=66$, $P<0.001$; genetics, $\chi^{2}(7)=41, P<0.001$; theory of mind, $\chi^{2}(7)=63, P<0.001$; neuroimaging, $\chi^{2}(7)=41, P<0.001$ ).

Immune research demonstrated a relatively large increase in the number of publications within the past 5 years (Figure 1b). However, unlike research on oxidative stress and toxicants, which had relatively few publications until 2000, research on immune dysfunction in ASD started in 1986 and has increased steadily since that time. The growth in immune research is similar to oxidative stress, toxicant exposure, genetic and neuroimaging, but different from the other areas (immune: mitochondrial dysfunction, $\chi^{2}(7)=31, P<0.001$; neuropathology, $\chi^{2}(7)=41, P<0.001$; theory of mind, $\chi^{2}(7)=36, P<0.001$ )

Genetic research started out with the largest number of papers in 1971-1975 and has grown since then, except for a small dip during 1991-1995. Because of this profile, the trend in publications in genetics was significantly different than for oxidative stress $\left(\chi^{2}(7)=28, P<0.001\right)$; mitochondrial dysfunction $\left(\chi^{2}(7)=39, \quad P<0.001\right) ; \quad$ toxicants $\quad\left(\chi^{2}(7)=41\right.$, $P<0.001)$; neuroimaging $\left(\chi^{2}(7)=32, P<0.001\right)$ and neuropathology $\left(\chi^{2}(7)=34, P<0.001\right)$.

Like genetic research, neuroimaging research started early, in 1976-1980, had a rapid rise, and then slowed down in the 1990s when interest in other areas of ASD research began to increase. Because of this profile, the trend in publications in neuroimaging research was significantly different than for oxidative stress $\left(\chi^{2}(7)=25, P<0.001\right)$; mitochondrial dysfunction $\left(\chi^{2}(7)=23, \quad P<0.001\right) ; \quad$ toxicants $\left(\chi^{2}(7)=41\right.$, $P<0.001)$; genetics $\left(\chi^{2}(7)=32, P<0.001\right)$ and neuropathology $\left(\chi^{2}(7)=22, P<0.001\right)$ publications.

Mitochondrial dysfunction probably has the most unique trend. As best seen in Figure 1c, a significant number of publications on mitochondrial function in ASD did not start until 1986-1990 and represent 11\% of publications during that 5-year period. From 1986-
1990 to 2001-2005, the absolute number of publications on mitochondrial dysfunction stayed relatively stable, resulting in this area of research representing proportionally less of the total number of studies on autism research in the eight areas. However, during the years 2006-2010, the number of studies on mitochondrial dysfunction increased considerably, resulting in recent growth in this area of research. Because of this unique profile, the trend in publications in the area of mitochondrial dysfunction was significantly different than for immune $\left(\chi^{2}(7)=31\right.$, $P<0.001)$; oxidative stress $\left(\chi^{2}(7)=24, P<0.001\right)$; toxicants $\quad\left(\chi^{2}(7)=44, \quad P<0.001\right)$; neuropathology $\left(\chi^{2}(7)=23, P<0.0001\right)$; theory of mind $\left(\chi^{2}(7)=21\right.$, $P<0.001)$ and genetic $\left(\chi^{2}(7)=39, P<0.001\right)$ research.

Like mitochondrial dysfunction, neuropathology research started with a relatively larger number of publications in 1986-1990, representing $8 \%$ of the publications during that 5-year period, and then demonstrated a stable number of absolute publications until 2001 when the number of publications increased and stabilized again. Because of the muted growth in this area of autism research relative to the other areas, it represents only $2 \%$ of the papers on ASD from 2006 to 2010. Because of this profile, the trend in publications in neuropathology research was significantly different than for immune $\left(\chi^{2}(7)=41\right.$, $P<0.001) ; \quad$ oxidative stress $\left(\chi^{2}(7)=43, \quad P<0.001\right)$; mitochondrial dysfunction $\left(\chi^{2}(7)=23, P<0.001\right)$; toxicants $\left(\chi^{2}(7)=66, P<0.001\right)$; neuroimaging $\left(\chi^{2}(7)=22\right.$, $P<0.001)$ and genetics $\left(\chi^{2}(7)=34, P<0.001\right)$ research.

Theory of mind has steadily grown since the 19761980 period, but other areas of research have grown faster in the years 2006-2010, including immune, oxidative stress and mitochondrial dysfunction research, resulting in a significantly different profile for theory of mind (immune, $\chi^{2}(7)=36, P<0.001$; oxidative stress, $\chi^{2}(7)=43, P<0.001$; mitochondrial dysfunction, $\chi^{2}(7)=21, P<0.001$; toxicants, $\chi^{2}(7)=63$, $P<0.001)$

In summary, many areas of autism research have grown over the past 40 years, particularly research on immune dysfunction, oxidative stress, toxicant exposures, genetics and neuroimaging. Research on mitochondrial dysfunction has also grown over the past 40 years, but after an initial increase from 1986 to 1990, this area of research did not grow relative to other areas and has only recently started to demonstrate an increase in interest in the last 5 years. Certain areas of research appear to have declined in recent years relative to other areas of autism research, including theory of mind and neuropathology research. Overall, genetic research continues to account for the majority of autism research.

\section{Classification of studies within the four major research areas}

Immune dysregulation and inflammation in ASD. A total of 437 publications (see Supplementary Material for reference citations) investigated a relationship 
between immune dysregulation and/or inflammation and ASD or Rett syndrome, with 416 (95\%) implicating a relationship. Twenty-one publications ( $5 \%$, all of them clinical studies) failed to find an association between immune dysregulation or inflammation and ASD. Publications suggesting a relationship between immune dysregulation and/or inflammation and ASD either reported generalized or organ specific (for example, the brain or GI tract) immune dysregulation/inflammation. An association between ASD and generalized immune dysregulation/ inflammation was implicated in 320 publications $(73 \%)$, including 155 clinical studies, 91 review articles or hypothesis-generating papers, 34 studies using animal models, 19 publications on Rett syndrome and 21 clinical-treatment studies. Neuroinflammation or brain immune dysregulation were implicated in ASD in $65(15 \%)$ publications, including 52 clinical studies and 13 review articles. GI inflammation or immune dysregulation were implicated in ASD in $31(7 \%)$ publications, including 24 clinical studies and 7 review articles. Ninety-five publications (not included in the initial 437 publications) discussed a possible link between vaccines (mainly MMR vaccine) and immune dysregulation and/or inflammation (especially of the GI tract) in ASD; the majority of these 95 publications reported no significant association.

Of the 231 clinical studies (combined: 155 for generalized immune dysregulation, 52 for neuroinflammation and 24 for GI inflammation) and 21 treatments studies in ASD, $19(8 \%)$ met Level-1 evidence, $164(65 \%)$ were Level 2, $19(8 \%)$ were Level 3, $47(19 \%)$ were Level 4 and $3(1 \%)$ were Level 5. One-hundred ninety-four $(77 \%)$ studies were controlled and $58(23 \%)$ were uncontrolled. One-hundred eighty-four (73\%) studies reported biomarker abnormalities in ASD including 30 studies that reported cytokine abnormalities and 23 studies that reported the presence of autoantibodies to brain tissue. Twenty studies reported abnormal biomarkers of immune function in brain tissue (16 studies) or cerebrospinal fluid (4 studies). Nine studies reported abnormalities in certain growth factors, including epidermal growth factor, hepatocyte growth factor, transforming growth factor-beta 1, insulin-like growth factor, platelet-derived growth factor, and nerve growth factor. See Table 3 for additional abnormal biomarker measurements. Eighteen studies reported that biomarkers of inflammation or immune dysregulation were positively correlated to the severity of ASD. Ten studies reported an increased prevalence of autoimmune disorders in family members of children with ASD. Thirteen studies reported allergies in children with ASD and 13 implicated infections in ASD. Six studies suggested that immune abnormalities or inflammation were related to mitochondrial dysfunction, oxidative stress or exposure to toxicants.

Oxidative stress in ASD. A total of 115 publications (see Supplementary Material for reference citations)
Table 3 Abnormalities in biomarkers of immune dysregulation or inflammation in ASD

$\begin{array}{ll}\text { Abnormality } & \begin{array}{l}\text { Number of } \\ \text { studies }\end{array}\end{array}$

Cytokine abnormalities 30

Autoantibodies to brain tissue

23

Abnormal brain or CSF biomarkers

Abnormal number of CD4 or CD8 cells

Alterations in MHC

Antibodies to foods

Abnormalities in immunoglobulins

Abnormalities in certain growth factors

Genetic mutations affecting immune

function

Maternal antibodies to fetal brain tissue or

lymphocytes

Decreased Bcl-2 expression

Alterations in BDNF

Microglial activation

Abnormalities in natural killer cells

Abnormal leptin levels

20

16

15

12

11

9

7

7

6

6

3

3

2

Abbreviations: ASD, autism spectrum disorders; BDNF, brain-derived neurotrophic factor; CSF, cerebrospinal fluid; MHC, major histocompatibility complex.

investigated a relationship between oxidative stress and ASD or Rett syndrome, with all $115(100 \%)$ suggesting an association. These publications included 51 clinical studies, 41 review articles or hypothesis-generating papers, 7 clinical-treatment studies, 6 publications reporting animal models and 10 publications on Rett syndrome.

Of the 51 clinical and 7 treatment studies in ASD, 3 (5\%) met Level-1 evidence, 37 (64\%) were Level 2, 12 $(21 \%)$ were Level 3, $6(10 \%)$ were Level 4 and none were Level 5. Forty-three (74\%) studies were controlled and $15(26 \%)$ were uncontrolled. Fifty-one $(88 \%)$ of these studies reported abnormal biomarkers of oxidative stress, including alterations in glutathione (16 studies), vitamin E (3 studies), tetrahydrobiopterin (4 studies) and antioxidant enzyme levels, such as glutathione peroxidase, paraoxonase and superoxide dismutase (11 studies). Eight studies reported abnormal biomarkers of lipid peroxidation including four that measured isoprostane levels. Four studies reported increased oxidative stress in postmortem brain tissue. Eight studies reported mutations in antioxidant enzymes or pathways, such as glutathione peroxidase, superoxide dismutase, glutathione-S-transferase, delta aminolevulinic acid dehydratase, methylenetetrahydrofolate reductase, catechol-O-methyltransferase and the reduced folate carrier. The seven treatment studies reported various clinical and/or biochemical improvements with injected methylcobalamin and oral folinic acid, tetrahydrobiopterin, dimercaptosuccinic acid, betaine and vitamin $\mathrm{E}$ administration. Five studies reported a positive correlation between biomarkers of greater oxidative stress and the severity of ASD. Twenty studies reported a potential link between oxidative 
stress and mitochondrial dysfunction, inflammation and/or environmental toxicant exposures.

Mitochondrial dysfunction in ASD. A total of 153 publications (see Supplementary Material for reference citations) investigated a relationship between mitochondrial dysfunction and ASD or Rett syndrome, with $145(95 \%)$ suggesting a relationship. Eight publications (5\%, all of them clinical studies) failed to find an association between mitochondrial dysfunction and ASD (7 studies) or Rett syndrome (one study). The 145 publications implicating an association between mitochondrial dysfunction and ASD included 63 clinical studies, 22 review articles or hypothesis-generating papers, 11 publications reporting animal models and 49 studies of Rett syndrome.

Of the 63 clinical studies in ASD, $14(22 \%)$ met Level-1 evidence, 16 (25\%) were Level 2, 2 (3\%) were Level 3, 31 were Level 4 (49\%) and none were level 5. Thirty-four (54\%) studies were controlled and 29 $(46 \%)$ were uncontrolled. Fifty-three $(84 \%)$ of these studies reported abnormal biomarkers, which could relate to mitochondrial dysfunction. Commonly abnormal biomarkers included lactic acid (18 studies), pyruvate (11 studies), carnitine (7 studies) and plasma amino acids (4 studies). Twenty-two studies reported at least one abnormal electron transport chain finding. Five studies reported evidence of mitochondrial dysfunction as measured by in vitro mitochondrial activity from lymphocytes obtained from individuals with ASD. Twenty studies reported an association between a genetic abnormality and mitochondrial dysfunction in ASD. Two studies reported a positive correlation between biomarkers of mitochondrial dysfunction and the severity of ASD. Four studies noted a potential overlap between mitochondrial dysfunction and environmental toxicant exposures, oxidative stress and inflammation.

Environmental toxicant exposures in ASD. A total of 190 publications (see Supplementary Material for reference citations) investigated a relationship between environmental toxicant exposures (such as pesticides, chemicals, phthalates, polychlorinated biphenyls, solvents, heavy metals or other pollutants) and ASD, with 170 (89\%) implicating a relationship and $20(11 \%)$ publications (19 clinical studies and 1 letter to the editor) failing to find an association between environmental toxicant exposures and ASD. The 170 publications suggesting an association between environmental toxicant exposures and ASD included 71 clinical studies, 67 review articles or hypothesis-generating papers, 26 publications of animal models and 6 treatment studies. Forty-seven publications (not included in the initial 190 publications) examined a possible association between thimerosal (ethylmercury) in vaccines and ASD, with 37 publications reporting no significant association and 10 (all but one publication were from the same authors) reporting some type of association. No publications reported an association or lack of an association between environmental toxicant exposures and Rett syndrome.

Of the 71 clinical and 6 treatment studies in ASD, 9 (12\%) met Level 1 criteria, 35 (45\%) were Level 2, 14 $(18 \%)$ were Level 3, 19 (25\%) were Level 4 and none were Level 5. Fifty (65\%) studies were controlled and $27(35 \%)$ were uncontrolled. Nineteen of these studies implicated lead exposure in ASD, 25 implicated mercury exposure, 8 studies implicated exposure to other toxic metals, 18 implicated exposure to chemicals, pesticides or other pollutants and 4 implicated exposure to dietary-related toxicants. Ten studies reported urinary porphyrins were higher in children with ASD compared with controls or laboratory reference ranges. Four studies implicated genetic mutations that might impair detoxification, and eight studies suggested that children with ASD may have a decreased ability to eliminate toxicants. Eight studies reported a positive association between exposure to toxicants and the severity of ASD. Six studies noted a potential overlap between environmental toxicant exposures and mitochondrial dysfunction, oxidative stress and inflammation.

\section{Discussion}

This article reviewed the trends in publications considering four physiological abnormalities potentially related to ASD: immune dysregulation/inflammation, oxidative stress, mitochondrial dysfunction and environmental toxicant exposures. The growth in these areas of research as well as the strength of the evidence presented was reviewed. These four areas were compared with four other well-known areas of ASD research. Below we outline some of the common themes in these four key areas of research.

\section{Trends in publications in four major areas}

Most publications (552 of 895, 62\%) in the four major areas were published in the last 5 years (2006-2010), with the great majority $(736,82 \%)$ published in the last 10 years (2001-2010). In general, there has been a precipitous rise in publications investigating these four key areas of physiological abnormalities within the last decade, with oxidative stress and exposure to toxicants growing the fastest and research on immune dysregulation/inflammation growing only slightly slower. Research on mitochondrial dysfunction started several decades ago but only grew slowly. Although research on mitochondrial dysfunction has grown in the last decade, such growth has not been as fast as these other key areas of ASD research. In comparison, other areas of research on ASD also continued to grow, especially genetic research, which represents the largest number of publications in ASD research for any given 5-year time period and continues to grow at a significant pace. Although neuroimaging continued to show significant growth over the last decade, growth in neuropathology and theory of mind research has not kept up with these 
other research areas in the last decade. Thus, overall it appears that areas of research relating to physiological abnormalities are growing considerably within the last decade at a rate similar to other, more traditional, thriving areas of ASD research (for example, genetics). It does appear that these new areas of research on the physiological abnormalities related to ASD may be replacing research efforts in older areas of ASD research, such as theory of mind and neuropathology.

The identified studies provide strong evidence for an association between these physiological abnormalities and ASD as the great majority of the studies reported an association. The number of published studies documenting this association has significantly increased in the medical literature within the last 10 years as compared with the previous 30 years (see Table 1). Furthermore, the increase in studies finding an association is not due to previous studies not finding an association, but rather due to the fact that such associations are starting to garner more serious attention and are undergoing systematic investigations. In fact, as more studies examine the potential associations between these physiological abnormalities and ASD, the associations are being confirmed. Overall, our analysis supports the continuation of these potentially important areas of research in ASD as these studies are uncovering new and novel associations that can provide significant insight into the etiology of ASD.

\section{Strength of evidence in four major areas}

Although the number of manuscripts in the four major areas of research has grown considerably in recent years, the manuscript quality is just as important, if not more important, as quantity. In order to present a judgment of manuscript quality, we ranked all of the clinical and treatment publications identified using a validated level of evidence scale, ${ }^{52}$ which ranged from level 1 (strongest evidence) to level 5 (weakest evidence). Overall, most of the manuscripts identified within the four major areas of research were rated as strong studies (for example, level 1 or level 2). Over two-thirds of the manuscripts discussing evidence for immune dysregulation/ inflammation $(73 \%)$ and oxidative stress $(69 \%)$ were level 1 or level 2, whereas studies on environmental toxicants $(57 \%)$ and mitochondrial dysfunction $(47 \%)$ demonstrated a lower percentage of very strong studies. The fact that not all studies were of very strong caliber should not be unexpected in new areas of research. For example, for mitochondrial dysfunction, many of the studies were either single-case reports or case series (level 4). However, in order for a new clinical entity (such as mitochondrial dysfunction in ASD) to be recognized, it is common for case reports to be published to introduce and confirm a new clinical entity before sufficient interest is generated to commit resources to completing larger high-quality, stronger studies. Overall, given the fact that these are recently evolving areas of research, the fact that over half of the papers are strong provides excellent evidence that these areas of research are growing in quality as well as quantity. However, additional studies in environmental toxicant exposures and mitochondrial dysfunction in ASD are warranted.

\section{Overlaps between four major areas}

A total of 36 publications reported a potential link between oxidative stress (20 publications), mitochondrial dysfunction (4 publications), immune dysregulation/inflammation (6 publications) and/or environmental toxicants (6 studies). At this point, it is unclear what triggers immune dysregulation, inflammation, oxidative stress and/or mitochondrial dysfunction in some individuals with ASD. With the interconnections between these physiological abnormalities, it is very possible that one physiological abnormality could trigger other physiological abnormalities. For example, environmental toxicant exposures can induce immune dysregulation and/or inflammation, ${ }^{53-57}$ oxidative stress ${ }^{58-62}$ and mitochondrial dysfunction. ${ }^{63-69}$ Thus, environmental toxicant exposure is a ripe area of research for triggering additional physiological abnormalities. Overall, it is possible that the interrelationships between these physiological abnormalities could result in an adversarial relationship such that one physiological abnormality could trigger other physiological abnormalities, which could reinforce the first, resulting in a downward spiral that could result in severe metabolic derangements. ${ }^{4}$ Additional studies are needed to consider the interrelationships between the physiological abnormalities examined in this study. Further research into these areas may also provide insight into additive effect of abnormalities in critical physiological processes that contribute to ASD and other psychiatric disorders.

\section{Integrating these novel areas of research with more traditional research areas}

To date, many genetic studies have concentrated on single genes that might cause autism. However, it is possible that integrating an understanding of the physiological abnormalities reviewed in this paper with genetic findings may lead to a better understanding of how genetic polymorphisms interact with the environment to contribute to psychiatric disorders. This may also provide a deeper understanding of the pathophysiological mechanisms that cause these disorders.

The relationship of the four major areas to understanding the etiology and treatment of ASD As 33 studies reported an association between the severity of ASD and immune dysregulation/inflammation (18 studies), oxidative stress (5 studies), mitochondrial dysfunction (2 studies) or toxicant exposures (8 studies), it is possible these physiological abnormalities contribute to the etiology of autism. However, the 33 studies reported these four physiological abnormalities in children with ASD after the 
diagnosis of ASD was made. Therefore, it is unclear if these physiological abnormalities existed in children with ASD before they developed ASD or if these abnormalities had a causal role in the development of ASD. If they do have a causal role, then it would be expected that these abnormalities would be significantly associated with the severity of ASD, as noted in these 33 studies. Given these findings, it is possible that the identification and treatment of these abnormalities before the development of ASD might prevent ASD or at least reduce the severity of ASD, once acquired. Additional studies are needed to know whether or not immune dysregulation/inflammation, oxidative stress, mitochondrial dysfunction or environmental toxicant exposures contribute to the development of ASD or if they are merely epiphenomena or comorbidities.

If these physiological abnormalities are shown to contribute to ASD development, then it is possible that treatment of these abnormalities may lead to clinical improvements in ASD symptoms or behaviors. As such, several reviewed publications reported various clinical improvements in some children with ASD using treatments that targeted immune dysregulation, inflammation, toxicity and/or oxidative stress. ${ }^{70-93}$ Additional studies are needed to investigate if treatment of these physiological abnormalities improves the symptomatology of ASD.

Several reviewed publications discussed the use of biomarkers to identify immune dysregulation, inflammation, oxidative stress, mitochondrial dysfunction or environmental toxicant exposures in individuals with ASD. ${ }^{77,94-102}$ The use of biomarkers to identify these physiological abnormalities may help lead to new treatment strategies for individuals with ASD and also help segregate ASD children into subgroups $^{103}$ that may respond differently to various treatments. Further studies are needed to examine these possibilities.

\section{Contributions of four major areas to other areas of psychiatric disorders}

Abnormalities in the four major areas reviewed in this articles have been reported to contribute to other psychiatric disorders, including schizophrenia, ${ }^{18,29,36,37,44-47}$ bipolar disorder, ${ }^{19,39,40,44-46}$ depression, ${ }^{20,28,38,45,46}$ dementia, ${ }^{45,46}$ Alzheimer's disease ${ }^{17,41}$ and ADHD. ${ }^{25-27}$ Additional studies of these physiological abnormalities and their effects on certain metabolic systems may lead to new insights into the pathophysiology of ASD and other psychiatric disorders, and may help in the understanding of whether there are pathophysiological processes that are common to many psychiatric disorders.

\section{Limitations}

This review had some limitations. We did not undertake a thorough review of each publication to determine if any of the publications explained how or why the reported physiological abnormality was associated with ASD; instead, we only collated whether or not the reviewed abnormality was implicated or not implicated in ASD. A systematic review of each of these physiological abnormalities, where strengths and weakness of included studies are discussed and additional areas of needed research identified would be very helpful in understanding these physiological abnormalities in terms of how they contribute to ASD. For example, recently we published a systematic review and meta-analysis concerning mitochondrial dysfunction in ASD. ${ }^{4}$

Another limitation of this review was that some studies reporting a negative association between ASD and immune dysregulation/inflammation, oxidative stress, mitochondrial dysfunction and/or environmental toxicant exposures may not have been published because of negative findings (a form of publication bias). Finally, we only examined a small percentage of published studies on ASD. The 906 unique studies that examined the four major areas in ASD only comprise approximately $6 \%(906 / 16310)$ of the studies concerning ASD published in the last 40 years.

\section{Conclusions}

A large percentage of publications implicated an association between ASD and immune dysregulation/inflammation, oxidative stress, mitochondrial dysfunction and toxicant exposures. The strongest evidence was for immune dysregulation/inflammation and oxidative stress followed by toxicant exposures and mitochondrial dysfunction. In all areas, at least $45 \%$ of the publications were rated as providing strong evidence for an association between the physiological abnormalities and ASD. Most publications in the four major areas were published in the last 5 years. The largest relative growth was in immune dysregulation/inflammation, oxidative stress, toxicant exposures, genetics and neuroimaging. Research on mitochondrial dysfunction started growing in the last 5 years. Theory of mind and neuropathology research has declined in recent years. Although most publications implicated an association between the four major areas and ASD, publication bias may have led to an overestimation of this association. Further research into these physiological areas may provide insight into general or subsetspecific processes that could contribute to the development of ASD and other psychiatric disorders.

\section{Conflict of interest}

Daniel Rossignol has two children with ASD and is a practicing primary-care physician who treats ASD children with standard and integrative treatments. He has received funding from the International Hyperbarics Association for two studies on the use of hyperbaric treatment in children with autism. ${ }^{88,104} \mathrm{He}$ was also a coauthor of a case report of spironolactone use in a child with autism. ${ }^{82}$ He has no additional competing interests. Richard Frye declares no conflict of interest. 


\section{Acknowledgements}

This research was supported by the Autism Research Institute.

\section{References}

1 APA. Diagnostic and Statistical Manual of Mental Disorders, 4th edn. American Psychiatric Association: Washington, DC, 1994.

2 Rice C. Prevalence of autism spectrum disorders - Autism and Developmental Disabilities Monitoring Network, United States, 2006. MMWR Surveill Summ 2009; 58: 1-20.

3 Schaefer GB, Mendelsohn NJ. Genetics evaluation for the etiologic diagnosis of autism spectrum disorders. Genet Med 2008; 10: 4-12.

4 Rossignol DA, Frye RE. Mitochondrial dysfunction in autism spectrum disorders: a systematic review and meta-analysis. Mol Psychiatry 2011.

5 Buie T, Campbell DB, Fuchs III GJ, Furuta GT, Levy J, Vandewater $\mathrm{J}$ et al. Evaluation, diagnosis, and treatment of gastrointestinal disorders in individuals with ASDs: a consensus report. Pediatrics 2010; 125(Suppl 1): S1-18.

6 James SJ, Melnyk S, Jernigan S, Cleves MA, Halsted CH, Wong DH et al. Metabolic endophenotype and related genotypes are associated with oxidative stress in children with autism. Am J Med Genet B Neuropsychiatr Genet 2006; 141: 947-956.

7 Ashwood P, Krakowiak P, Hertz-Picciotto I, Hansen R, Pessah I, Van de Water J. Elevated plasma cytokines in autism spectrum disorders provide evidence of immune dysfunction and are associated with impaired behavioral outcome. Brain Behav Immun 2010; 25: 40-45.

8 Ming X, Brimacombe M, Chaaban J, Zimmerman-Bier B, Wagner GC. Autism spectrum disorders: concurrent clinical disorders. J Child Neurol 2008; 23: 6-13.

9 Herbert MR. Autism: a brain disorder or a disorder that affects the brain. Clinical Neuropsychiatry 2005; 2: 354-379.

10 Harrison PJ, Weinberger DR. Schizophrenia genes, gene expression, and neuropathology: on the matter of their convergence. Mol Psychiatry 2005; 10: 40-68;, image 45.

11 Kleinman JE, Law AJ, Lipska BK, Hyde TM, Ellis JK, Harrison PJ et al. Genetic neuropathology of schizophrenia: new approaches to an old question and new uses for postmortem human brains. Biol Psychiatry 2011; 69: 140-145.

12 Shao L, Martin MV, Watson SJ, Schatzberg A, Akil H, Myers RM et al. Mitochondrial involvement in psychiatric disorders. Ann Med 2008; 40: 281-295.

13 Ng F, Berk M, Dean O, Bush AI. Oxidative stress in psychiatric disorders: evidence base and therapeutic implications. Int $J$ Neuropsychopharmacol 2008; 11: 1-26.

14 Dantzer R, O'Connor JC, Freund GG, Johnson RW, Kelley KW. From inflammation to sickness and depression: when the immune system subjugates the brain. Nat Rev Neurosci 2008; 9: 46-56.

15 Burke MG, Miller MD. Practical guidelines for evaluating lead exposure in children with mental health conditions: molecular effects and clinical implications. Postgrad Med 2011; 123: 160-168.

16 Streit WJ, Mrak RE, Griffin WS. Microglia and neuroinflammation: a pathological perspective. J Neuroinflammation 2004; 1: 14.

17 Eikelenboom P, Bate C, Van Gool WA, Hoozemans JJ, Rozemuller JM, Veerhuis R et al. Neuroinflammation in Alzheimer's disease and prion disease. Glia 2002; 40: 232-239.

18 Doorduin J, de Vries EF, Willemsen AT, de Groot JC, Dierckx RA, Klein HC. Neuroinflammation in schizophrenia-related psychosis: a PET study. J Nucl Med 2009; 50: 1801-1807.

19 Rao JS, Harry GJ, Rapoport SI, Kim HW. Increased excitotoxicity and neuroinflammatory markers in postmortem frontal cortex from bipolar disorder patients. Mol Psychiatry 2010; 15: 384-392.

20 Dobos N, Korf J, Luiten PG, Eisel UL. Neuroinflammation in Alzheimer's disease and major depression. Biol Psychiatry 2010; 67: 503-504.
21 Pardo CA, Vargas DL, Zimmerman AW. Immunity, neuroglia and neuroinflammation in autism. Int Rev Psychiatry 2005; 17: 485-495.

22 Li X, Chauhan A, Sheikh AM, Patil S, Chauhan V, Li XM et al. Elevated immune response in the brain of autistic patients. J Neuroimmunol 2009; 207: 111-116.

$23 \mathrm{Li}$ Z, Dong T, Proschel C, Noble M. Chemically diverse toxicants converge on Fyn and c-Cbl to disrupt precursor cell function. PLOS Biol 2007; 5: e35.

24 Grandjean P, Landrigan PJ. Developmental neurotoxicity of industrial chemicals. Lancet 2006; 368: 2167-2178.

25 Braun JM, Kahn RS, Froehlich T, Auinger P, Lanphear BP. Exposures to environmental toxicants and attention deficit hyperactivity disorder in US children. Environ Health Perspect 2006; 114: 1904-1909.

26 Nigg JT, Knottnerus GM, Martel MM, Nikolas M, Cavanagh K, Karmaus $\mathrm{W}$ et al. Low blood lead levels associated with clinically diagnosed attention-deficit/hyperactivity disorder and mediated by weak cognitive control. Biol Psychiatry 2008; 63: 325-331.

27 Bouchard MF, Bellinger DC, Wright RO, Weisskopf MG. Attention-deficit/hyperactivity disorder and urinary metabolites of organophosphate pesticides. Pediatrics 2010; 125: e1270-e1277.

28 Amr MM, Halim ZS, Moussa SS. Psychiatric disorders among Egyptian pesticide applicators and formulators. Environ Res 1997; 73: 193-199.

29 Opler MG, Brown AS, Graziano J, Desai M, Zheng W, Schaefer C et al. Prenatal lead exposure, delta-aminolevulinic acid, and schizophrenia. Environ Health Perspect 2004; 112: 548-552.

30 Palmer RF, Blanchard S, Wood R. Proximity to point sources of environmental mercury release as a predictor of autism prevalence. Health Place 2009; 15: 18-24.

31 Windham GC, Zhang L, Gunier R, Croen LA, Grether JK. Autism spectrum disorders in relation to distribution of hazardous air pollutants in the San Francisco bay area. Environ Health Perspect 2006; 114: 1438-1444.

32 Roberts EM, English PB, Grether JK, Windham GC, Somberg L, Wolff C. Maternal residence near agricultural pesticide applications and autism spectrum disorders among children in the California Central Valley. Environ Health Perspect 2007; 115: 1482-1489.

33 Dhalla NS, Temsah RM, Netticadan T. Role of oxidative stress in cardiovascular diseases. J Hypertens 2000; 18: 655-673.

34 Maritim AC, Sanders RA, Watkins III JB. Diabetes, oxidative stress, and antioxidants: a review. J Biochem Mol Toxicol 2003; 17: 24-38.

35 de Champlain J, Wu R, Girouard H, Karas M, EL Midaoui A, Laplante MA et al. Oxidative stress in hypertension. Clin Exp Hypertens 2004; 26: 593-601.

36 Prabakaran S, Swatton JE, Ryan MM, Huffaker SJ, Huang JT, Griffin JL et al. Mitochondrial dysfunction in schizophrenia: evidence for compromised brain metabolism and oxidative stress. Mol Psychiatry 2004; 9: 643 684-697.

37 Tosic M, Ott J, Barral S, Bovet P, Deppen P, Gheorghita F et al. Schizophrenia and oxidative stress: glutamate cysteine ligase modifier as a susceptibility gene. Am J Hum Genet 2006; 79: 586-592.

38 Ozcan ME, Gulec M, Ozerol E, Polat R, Akyol O. Antioxidant enzyme activities and oxidative stress in affective disorders. Int Clin Psychopharmacol 2004; 19: 89-95.

39 Andreazza AC, Kauer-Sant'anna M, Frey BN, Bond DJ, Kapczinski F, Young LT et al. Oxidative stress markers in bipolar disorder: a meta-analysis. J Affect Disord 2008; 111: 135-144.

40 Frey BN, Andreazza AC, Kunz M, Gomes FA, Quevedo J, Salvador $\mathrm{M}$ et al. Increased oxidative stress and DNA damage in bipolar disorder: a twin-case report. Prog Neuropsychopharmacol Biol Psychiatry 2007; 31: 283-285.

41 Emerit J, Edeas M, Bricaire F. Neurodegenerative diseases and oxidative stress. Biomed Pharmacother 2004; 58: 39-46.

42 Chauhan A, Chauhan V. Oxidative stress in autism. Pathophysiology 2006; 13: 171-181.

43 Haas RH, Parikh S, Falk MJ, Saneto RP, Wolf NI, Darin N et al. Mitochondrial disease: a practical approach for primary care physicians. Pediatrics 2007; 120: 1326-1333. 
44 Clay HB, Sillivan S, Konradi C. Mitochondrial dysfunction and pathology in bipolar disorder and schizophrenia. Int $J$ Dev Neurosci 2011; 29: 311-324.

45 Kato M, Nakamura M, Ichiba M, Tomiyasu A, Shimo H, Higuchi I et al. Mitochondrial DNA deletion mutations in patients with neuropsychiatric symptoms. Neurosci Res 2011; 69: 331-336.

46 Scaglia F. The role of mitochondrial dysfunction in psychiatric disease. Dev Disabil Res Rev 2010; 16: 136-143.

47 Verge B, Alonso Y, Valero J, Miralles C, Vilella E, Martorell L. Mitochondrial DNA (mtDNA) and schizophrenia. Eur Psychiatry 2011; 26: 45-56.

48 Rossignol DA, Bradstreet JJ. Evidence of mitochondrial dysfunction in autism and implications for treatment. Am J Biochem Biotech 2008; 4: 208-217.

49 Frye RE, Rossignol DA. Mitochondrial dysfunction can connect the diverse medical symptoms associated with autism spectrum disorders. Pediatr Res 2011; 69(5 Part 2): 41R-47R.

50 Anderson MP, Hooker BS, Herbert MR. Bridging from cells to cognition in autism pathophysiology: biological pathways to defective brain function and plasticity. Am J Biochem Biotech 2008; 4: 167-176.

51 Roberts RA, Laskin DL, Smith CV, Robertson FM, Allen EM, Doorn JA et al. Nitrative and oxidative stress in toxicology and disease. Toxicol Sci 2009; 112: 4-16.

52 The Oxford 2011 Levels of Evidence. http://www.cebm.net/ index.aspx?o = 5653, (accessed 2011).

53 Sterzl I, Prochazkova J, Hrda P, Bartova J, Matucha P, Stejskal VD. Mercury and nickel allergy: risk factors in fatigue and autoimmunity. Neuro Endocrinol Lett 1999; 20: 221-228.

54 Kempuraj D, Asadi S, Zhang B, Manola A, Hogan J, Peterson E et al. Mercury induces inflammatory mediator release from human mast cells. J Neuroinflammation 2010; 7: 20.

55 Calderon-Garciduenas L, Reed W, Maronpot RR, Henriquez-Roldan C, Delgado-Chavez R, Calderon-Garciduenas A et al. Brain inflammation and Alzheimer's-like pathology in individuals exposed to severe air pollution. Toxicol Pathol 2004; 32: 650-658.

56 Havarinasab S, Hultman P. Organic mercury compounds and autoimmunity. Autoimmun Rev 2005; 4: 270-275.

57 Calderon-Garciduenas L, Solt AC, Henriquez-Roldan C, TorresJardon R, Nuse B, Herritt L et al. Long-term air pollution exposure is associated with neuroinflammation, an altered innate immune response, disruption of the blood-brain barrier, ultrafine particulate deposition, and accumulation of amyloid beta-42 and alphasynuclein in children and young adults. Toxicol Pathol 2008; 36: 289-310.

58 Yee S, Choi BH. Oxidative stress in neurotoxic effects of methylmercury poisoning. Neurotoxicology 1996; 17: 17-26.

59 Flora SJ. Arsenic-induced oxidative stress and its reversibility following combined administration of $\mathrm{N}$-acetylcysteine and meso 2,3-dimercaptosuccinic acid in rats. Clin Exp Pharmacol Physiol 1999; 26: 865-869.

60 Flora SJ, Pande M, Kannan GM, Mehta A. Lead induced oxidative stress and its recovery following co-administration of melatonin or N-acetylcysteine during chelation with succimer in male rats. Cell Mol Biol 2004; 50: OL543-OL551.

61 Hiura TS, Kaszubowski MP, Li N, Nel AE. Chemicals in diesel exhaust particles generate reactive oxygen radicals and induce apoptosis in macrophages. I Immunol 1999; 163: 5582-5591.

62 Bhadauria S, Flora SJ. Response of arsenic-induced oxidative stress, DNA damage, and metal imbalance to combined administration of DMSA and monoisoamyl-DMSA during chronic arsenic poisoning in rats. Cell Biol Toxicol 2007; 23: 91-104.

63 Mailloux R, Lemire J, Appanna V. Aluminum-induced mitochondrial dysfunction leads to lipid accumulation in human hepatocytes: a link to obesity. Cell Physiol Biochem 2007; 20: 627-638.

64 Fowler BA, Woods JS. Ultrastructural and biochemical changes in renal mitochondria during chronic oral methyl mercury exposure: the relationship to renal function. Exp Mol Pathol 1977; 27: 403-412.

65 Shenker BJ, Guo TL, O I, Shapiro IM. Induction of apoptosis in human T-cells by methyl mercury: temporal relationship between mitochondrial dysfunction and loss of reductive reserve. Toxicol Appl Pharmacol 1999; 157: 23-35.

66 Goyer RA. Toxic and essential metal interactions. Annu Rev Nutr 1997; 17: 37-50.

67 Pourahmad J, Mihajlovic A, O’Brien PJ. Hepatocyte lysis induced by environmental metal toxins may involve apoptotic death signals initiated by mitochondrial injury. Adv Exp Med Biol 2001; 500: 249-252.

68 Wong PW, Garcia EF, Pessah IN. ortho-substituted PCB95 alters intracellular calcium signaling and causes cellular acidification in PC12 cells by an immunophilin-dependent mechanism. J Neurochem 2001; 76: 450-463.

69 Yamano T, Morita S. Effects of pesticides on isolated rat hepatocytes, mitochondria, and microsomes II. Arch Environ Contam Toxicol 1995; 28: 1-7.

70 Stefanatos GA, Grover W, Geller E. Case study: corticosteroid treatment of language regression in pervasive developmental disorder. J Am Acad Child Adolesc Psychiatry 1995; 34: 1107-1111.

71 Komori H, Matsuishi T, Yamada S, Yamashita Y, Ohtaki E, Kato H. Cerebrospinal fluid biopterin and biogenic amine metabolites during oral R-THBP therapy for infantile autism. J Autism Dev Disord 1995; 25: 183-193.

72 James SJ, Melnyk S, Fuchs G, Reid T, Jernigan S, Pavliv O et al. Efficacy of methylcobalamin and folinic acid treatment on glutathione redox status in children with autism. Am J Clin Nutr 2009; 89: 425-430.

73 Dolske MC, Spollen J, McKay S, Lancashire E, Tolbert L. A preliminary trial of ascorbic acid as supplemental therapy for autism. Prog Neuropsychopharmacol Biol Psychiatry 1993; 17: 765-774.

74 Boris M, Goldblatt A, Edelson SM. Improvement in children with autism treated with intravenous gamma globulin. I Nutritional Environmental Medicine 2005; 15: 169-176.

75 Adams JB, Baral M, Geis E, Mitchell J, Ingram J, Hensley A et al. Safety and efficacy of oral DMSA therapy for children with autism spectrum disorders: part A-medical results. BMC Clin Pharmacol 2009; 9: 16.

76 Adams JB, Baral M, Geis E, Mitchell J, Ingram J, Hensley A et al. Safety and efficacy of oral DMSA therapy for children with autism spectrum disorders: part B - behavioral results. BMC Clin Pharmacol 2009; 9: 17.

77 Bertoglio K, Jill James S, Deprey L, Brule N, Hendren RL. Pilot study of the effect of methyl B12 treatment on behavioral and biomarker measures in children with autism. J Altern Complement Med 2010; 16: 555-560.

78 O'Hara NH, Szakacs GM. The recovery of a child with autism spectrum disorder through biomedical interventions. Altern Ther Health Med 2008; 14: 42-44.

79 Akhondzadeh S, Fallah J, Mohammadi MR, Imani R, Mohammadi M, Salehi B et al. Double-blind placebo controlled trial of pentoxifylline added to risperidone: effects on aberrant behavior in children with autism. Prog Neuropsychopharmacol Biol Psychiatry 2010; 34: 32-36.

80 Boris M, Kaiser CC, Goldblatt A, Elice MW, Edelson SM, Adams JB et al. Effect of pioglitazone treatment on behavioral symptoms in autistic children. J Neuroinflammation 2007; 4: 3 .

81 Bouvard MP, Leboyer M, Launay JM, Recasens C, Plumet MH, Waller-Perotte D et al. Low-dose naltrexone effects on plasma chemistries and clinical symptoms in autism: a double-blind, placebo-controlled study. Psychiatry Res 1995; 58: 191-201.

82 Bradstreet JJ, Smith S, Granpeesheh D, El-Dahr JM, Rossignol D. Spironolactone might be a desirable immunologic and hormonal intervention in autism spectrum disorders. Med Hypotheses 2007; 68: 979-987.

83 Fudenberg HH. Dialysable lymphocyte extract (DLyE) in infantile onset autism: a pilot study. Biotherapy 1996; 9: 143-147.

84 Gupta S. Treatment of children with autism with intravenous immunoglobulin. I Child Neurol 1999; 14: 203-205.

85 Gupta S. Immunological treatments for autism. J Autism Dev Disord 2000; 30: 475-479.

86 Gupta S, Aggarwal S, Heads C. Dysregulated immune system in children with autism: beneficial effects of intravenous immune 
globulin on autistic characteristics. I Autism Dev Disord 1996; 26: 439-452.

87 Gupta S, Rimland B, Shilling PD. Pentoxifylline: brief review and rationale for its possible use in the treatment of autism. J Child Neurol 1996; 11: 501-504.

88 Rossignol DA, Rossignol LW, James SJ, Melnyk S, Mumper E. The effects of hyperbaric oxygen therapy on oxidative stress, inflammation, and symptoms in children with autism: an openlabel pilot study. BMC Pediatr 2007; 7: 36.

89 Scifo R, Cioni M, Nicolosi A, Batticane N, Tirolo C, Testa N et al. Opioid-immune interactions in autism: behavioural and immunological assessment during a double-blind treatment with naltrexone. Ann Ist Super Sanita 1996; 32: 351-359.

90 Shenoy S, Arnold S, Chatila T. Response to steroid therapy in autism secondary to autoimmune lymphoproliferative syndrome. J Pediatr 2000; 136: 682-687.

91 Bradstreet JJ, Geier DA, Kartzinel JJ, Adams JB, Geier MR. A case-control study of mercury burden in children with autistic spectrum disorders. J Amer Physicians Surg 2003; 8: 76-79.

92 Lonsdale D, Shamberger RJ, Audhya T. Treatment of autism spectrum children with thiamine tetrahydrofurfuryl disulfide: a pilot study. Neuro Endocrinol Lett 2002; 23: 303-308.

93 Patel K, Curtis LT. A comprehensive approach to treating autism and attention-deficit hyperactivity disorder: a prepilot study. J Altern Complement Med 2007; 13: 1091-1097.

94 Bent S, Hendren RL. Improving the prediction of response to therapy in autism. Neurotherapeutics 2010; 7: 232-240.

95 Bradstreet JJ, Smith S, Baral M, Rossignol DA. Biomarker-guided interventions of clinically relevant conditions associated with autism spectrum disorders and attention deficit hyperactivity disorder. Altern Med Rev 2010; 15: 15-32.

96 Al-Gadani Y, El-Ansary A, Attas O, Al-Ayadhi L. Metabolic biomarkers related to oxidative stress and antioxi- dant status in Saudi autistic children. Clin Biochem 2009; 42: 1032-1040.

97 Al-Mosalem OA, El-Ansary A, Attas O, Al-Ayadhi L. Metabolic biomarkers related to energy metabolism in Saudi autistic children. Clin Biochem 2009; 42: 949-957.

98 Faber S, Zinn GM, Kern II JC, Kingston HM. The plasma zinc/ serum copper ratio as a biomarker in children with autism spectrum disorders. Biomarkers 2009; 14: 171-180.

99 Geier DA, Geier MR. A prospective study of mercury toxicity biomarkers in autistic spectrum disorders. I Toxicol Environ Health A 2007; 70: 1723-1730.

100 Geier DA, Kern JK, Garver CR, Adams JB, Audhya T, Geier MR. A prospective study of transsulfuration biomarkers in autistic disorders. Neurochem Res 2009; 34: 386-393.

101 Geier DA, Kern JK, Garver CR, Adams JB, Audhya T, Nataf R et al. Biomarkers of environmental toxicity and susceptibility in autism. J Neurol Sci 2009; 280: 101-108.

102 Kern JK, Geier DA, Adams JB, Mehta JA, Grannemann BD, Geier MR. Toxicity biomarkers in autism spectrum disorder: a blinded study of urinary porphyrins. Pediatr Int 2011; 53: 147-153.

103 Hendren RL, Bertoglio K, Ashwood P, Sharp F. Mechanistic biomarkers for autism treatment. Med Hypotheses 2009; 73: 950-954.

104 Rossignol DA, Rossignol LW, Smith S, Schneider C, Logerquist S Usman A et al. Hyperbaric treatment for children with autism: a multicenter, randomized, double-blind, controlled trial. BMC Pediatr 2009; 9: 21

(c) This work is licensed under the Creative Commons Attribution-NonCommercialNo Derivative Works 3.0 Unported License. To view a copy of this license, visit http://creativecommons.org/licenses/by-nc-nd/3.0/

Supplementary Information accompanies the paper on the Molecular Psychiatry website (http://www.nature.com/mp) 